Chirurgia (2017) 112: 505-508

No. 5, September - October

Copyright@ Celsius

http://dx.doi.org/10.21614/chirurgia.112.5.505

\title{
Education for Emergency Surgery and Trauma
}

\author{
Jonathan Tilsed
}

Hull York Medical School, United Kingdom

Corresponding author:

Jonathan Tilsed, MD

Consultant Colorectal Surgeon

Honorary Senior Lecturer Hull York

Medical School

Chairman UEMS

Division of Emergency Surgery

Past President European Society

for Trauma and Emergency Surgery

E-mail: tilsed@doctors.org.uk
Received: 23.08.2017

Accepted: 10.09 .2017
The system of apprenticeship developed across Europe during the Middle Ages and lasted well into the $19^{\text {th }}$ century. Under this ancient process of on-the-job training an apprentice was bound by a contract (indenture) to serve a master for between five and seven years and in return the master would teach him his trade. It was also the practice in some crafts for the apprentice to pass between masters to increase his experience. This was an effective method of training craftsmen that endured for centuries. Its demise was not due to the emergence of better training methods, but the fall in demand for the skills themselves.

The traditional method of surgical training not only evolved from this, but continued in the same style long after the formal craft apprenticeships had ceased. Expansion in consultant numbers and the pursuit of ever more specialised practice has made this form of training untenable. Although demand for the skills has increased, the master craftsmen have been lost. This is most evident in the field of emergency surgery and trauma.

Despite the fact that the majority of inpatient surgical admissions are unplanned, resulting from either surgical emergencies or trauma, there are few institutions where the service is designed primarily around their needs. Priority is usually given to elective admissions. Perhaps as a result of this the organisation of emergency and trauma care surgery across Europe is highly varied: no two countries have an identical system.

Emergency and trauma care surgery is, in essence, a spectrum covering orthopaedic trauma, visceral trauma and non-trauma emergency general surgery. There are two main patterns of organisation: the orthopaedic and general surgeon model which deals well with bony trauma and emergency general surgery, but less well with visceral trauma, and the 
model of the trauma surgeon based on the German Unfallchirurg which deals well with bony and visceral trauma, but less well with non-trauma emergency surgery.

To add further complexity in to the mix approximately $20 \%$ of countries have adopted a highly specialised version of the orthopaedic and general surgeon model involving vascular, thoracic, plastic, neuro- and urological surgery. The past twenty years have also seen the development of sub-specialisation within orthopaedic surgery into upper limb, lower limb, hip, shoulder, hand, foot and ankle and pelvic surgeons. While this may be good for elective practice, the requirements of emergency and trauma care surgery are not always conveniently tissue or region specific. To provide effective care the emergency surgeon must be able to manage the whole patient.

The founding fathers of the National Health Service in the UK believed that it would reduce the prevalence of illness and disease in the population, create a fitter, more productive workforce and be effectively selffunding. This proved to be a costly miscalculation. As healthcare systems develop, rather than reducing the prevalence of illness and disease in the population they enable sick people to live longer with the result that they create an ageing population with a higher incidence of co-morbidities.

It is no longer sufficient for the providers of emergency and trauma care surgery to deal with the acute problem in isolation; this has to be managed in the context of pre-existing illness and, in some cases, significant $\mathrm{co}^{-}$ morbidities that may until that point have been undiagnosed and untreated. Moreover some of the commonest clinical presentations: fractures, jaundice, abdominal pain or vomiting may be the first manifestations of advanced malignancy in patients who require palliative care rather than heroic surgery.

These problems are of course not unique to emergency and trauma care surgery, but the main difference between elective and emergency surgery are the effects of acute injury, whether pathological or traumatic, and sepsis on the patient's physiology. Critical care with the intensive support of respiration, cardiac function, tissue and organ perfusion, renal function and nutrition is an essential part of emergency and trauma care surgery.

In 2003, concerned by the inability of surgeons to provide emergency care, the American College of Surgeons, together with the American Association for the Surgery of Trauma, the Eastern Association for the Surgery of Trauma and the Western Trauma Association began to develop a model of acute care surgery. This consists of emergency surgery, trauma surgery and critical care, and the first training programmes were launched in 2008. Following on from the development of acute care surgery in the United States, emergency general surgeons are beginning to emerge in Europe. Although growing, they are small in numbers and, unlike the Unfallchirurgs, have yet to produce a critical mass of specialists in this field.

To provide the level of care that these complex patients require is often best done by a multidisciplinary team. It is not reasonable to expect emergency and trauma care surgeons to be experts in all fields, but they should know enough about them to be able to lead the team, understand the issues and decide on the most appropriate treatment and sequence of interventions. They must understand diagnostic tests and interventional radiology and know the limitations of them. They must know what treatment options are available and, while they may not necessarily understand the risks, benefits and limitations of all of them, they should be able to consult appropriately to obtain this information. Surgeons should know their limitations and when to seek help at all stages of their careers.

Emergency and trauma care surgeons require an understanding of chronic disease and the effects of extremes of age, acute medical conditions, diagnostic tests, interventions, critical care, palliative care and rehabilitation. They should be able to work as part of team with mutual support and lead the team when appropriate. Above all they need to be good communicators.

So with such a wide syllabus, how do we 
teach it? Just as there is no one correct way to deliver emergency and trauma surgery, there is no single way to train for it. Both have to be done, and have to be done well.

The key to emergency and trauma surgery lies in good decision making. This has to be backed up by good technical skills, but the wrong operation, no matter how beautifully executed, is still the wrong operation. In turn, decision making has to be underpinned by knowledge.

Although true education is a lifelong process, there are different phases that a surgeon will pass through in his or her professional life. The early stages are characterised by the acquisition of knowledge and basic skills. This is difficult to achieve without context or perspective and it should be the duty of the trainer to provide these for the trainee. While self-directed learning should be encouraged, the trainee will need to know that the direction is correct. The trainee should develop a thirst for knowledge following the lead of inspirational teachers. The trainee must have access to information and the ability to retain it, or at least the relevant parts of it, for future reference. Trainees no longer have to rely on memory alone. There is a wealth of available information, much of which is easily accessible, which gives today's trainees a distinct advantage over previous generations. However, while we may be swamped with information, it is important to distinguish this from knowledge. Text books are usually out of date before they are published although newer on-line platforms should improve this. The ease of publication has created an explosion in the number of scientific and pseudo-scientific journals available. Added to this, the pressure to publish and the distortion created by the bias towards publication of positive results, which may not be reproducible, has resulted in an overwhelming volume of poor quality literature. There is so much, in fact, that it would take several centuries to read all the medical articles published in a single year. It should also be remembered that the loudest voice, the most prolific author or the greatest on-line presence is not necessarily the best. Trainees need to be taught critical thinking in order to separate the true facts from speculation and to try to see through bias.

Decision-making and critical thinking are skills that are developed by practice especially when performed in a relevant context. The opportunity to discuss clinical cases or procedures can be of educational benefit to both the trainer and trainee, particularly if this is done in an environment where the trainer challenges the decisions of the trainee, and the trainee is encouraged to challenge the decisions of the trainer. This method of education, often formalised as case based discussions, has been used by generations of surgeons to train their juniors in grand rounds, at the bedside, in lecture theatres or in meetings. It works better if it is not an intimidating experience for the trainee.

One of the difficulties in training emergency and trauma surgeons is the reduced exposure to patients and procedures compared to that of previous generations of surgeons. This can be improved by rotating the trainee between trainers and institutions to ensure an appropriate breadth and depth of experience. As procedures become less frequently performed it follows logically that the need to learn them also falls. Medicine will continue to evolve, and surgical procedures that are common now will become obsolete. As much as we might like to think that we know all the answers, there is much that we do not know. Undoubtedly in 100 years time, surgeons will look back at many aspects of current surgical practice in disbelief.

It is important that the practical skills taught are commensurate with the needs of the healthcare system in which the trainee will work. The trainee needs to become proficient in dealing with common problems and conditions, and have the knowledge and competence to make the right decisions for less common problems. Not all surgeons will practice in remote areas or on humanitarian missions, but those who do may need to be additionally skilled in performing emergency caesarean sections, hysterectomies for post partum haemorrhage and in the application of 
external fixation devices for fractures.

The traditional learning method of 'see one, do one, teach one' requires sufficient background skills and experience to be able to develop new skills rapidly. We all learn at different rates and the gradient of our learning curves will vary between individuals and procedures. We also do not learn as well when tired, stressed or overworked. It is unreasonable to expect the trainee to become proficient at a procedure with minimal exposure to it, when a trained surgeon is expected to undertake a minimum number of procedures in order to maintain competence. This is not a paradigm shift, just a realistic expectation.

Over recent years a vast educational armamentarium has been developed across different learning platforms. Surgeons now have access to a wealth of information including courses, simulators, on-line learning packages, forums that facilitate discussion and problem solving and numerous videos demonstrating operative procedures. Videos, courses, workshops, wet labs, cadaver labs and animal labs can all be used to enhance practical skills. Complex procedures can be broken down into a series of steps and the trainee taught and assessed on each of these as their skills development permits. When the trainee is considered sufficiently competent, they can move on to more complex steps in the procedure and new procedures. If the development of these competencies is accurately recorded the information can be used by a different trainer to facilitate subsequent training.

Newer methods of training have been introduced including work based assessments, direct observation of procedural skills, case based discussions, procedure based assessments and clinical evaluation exercises. Are they really that novel, or do they formalise and document what good clinical teachers have always done?

At the end of the day the aim is to produce good doctors. But good doctors come in a variety of forms. A 10-20 year lag time is too late for making interventions in time if we get it wrong. The training system should be based around the needs of the trainees, not the service and there must be effective oversight of the process. To run an educational programme that delivers quality, consistency and reproducibility requires a degree of supervision and management. While there is some truth in the mantra " if you can't measure it you can't manage it", it is important that parameters measured are the most appropriate, not simply the easiest or most convenient. For example: reflecting on interactions with patients can be a valuable learning exercise at any point in a surgeon's career. It is far easier to record the number of these than to assess their relevance. How many superficial considerations equate to one profound lifechanging reflection? An over-reliance on meeting targets risks missing the point of education and turning education into a tick box exercise. The European Union of Medical Specialists (UEMS) has developed Board Qualifications in Emergency Surgery (https:// www.uemssurg.org/divisions/emergencysurgery/ebsq) and Trauma Surgery (https:// www.uemssurg.org/divisions/traumasurgery/ebsq-examination) which can be used as tools for standard setting and development of emergency and trauma surgery at a national and international level.

In addition to the skills and knowledge listed above, emergency and trauma surgeons need personal traits that cannot be taught: the temperament to be able to work in a changing environment, and to stand up to scrutiny, and to have the resilience to cope when things go wrong. While the circumstances under which training occurs have changed significantly over recent decades, so too have the tools available to deliver it. We ask a lot of our future surgeons particularly in emergency and trauma surgery. The mediaeval master craftsmen took a close interest in the training of their apprentices and we, as surgical trainers, must do the same. 